International scientific and technical conference

DOI: $10.34185 / 1991-7848 . i t m m .2020 .01 .010$

\title{
АНАЛИТИЧЕСКИЕ МОДЕЛИ ПРЕВРАЩЕНИЯ АУСТЕНИТА С УЧЕТОМ ВНУТРЕННИХ НАПРЯЖЕНИЙ
}

Бобырь С.В. д.т.н., Борисенко А.Ю, к.т.н., Лошкарев Д.В. инж.

Институт черной металлургии им. З.И. Некрасова НАН Украины

Аннотация. Рассмотрены аналитические модели превращения аустенита в стали, учитывающие влияние напряженного состояния на скорость превращения при термообработке сталей. Предложены простые аналитические выражения для расчета скорости и количества образующегося мартенсита в зависимости от величины напряжения в $\gamma$-фазе.

КЛючевЫе слова: ПРЕВРАЩЕНИЕ АУСТЕНИТА, МАРТЕНСИТ, АНАЛИТИЧЕСКИЕ МОДЕЛИ, ВНУТРЕННИЕ НАПРЯЖЕНИЯ, ТЕРМИЧЕСКАЯ ОБРАБОТКА.

Современное состояние вопроса. В области моделирования кинетики фазовых превращений наиболее фундаментальные исследования были проведены в работах А.Н. Колмогорова и М. Аврами, которые описывают аналитические уравнения, где объем вновь образуемой фазы ставится в зависимость от вероятности зарождения центров, линейной скорости их роста и прошедшего времени [1, 2].

Фундаментальные обзоры математического моделирования процесса фазовых превращений представлен в работах [3 - 5], в которых указано, что существующие аналитические модели не учитывают явно процесс нестационарного зарождения, а это противоречит физике процесса. Также отмечено, что аналитические модели не позволяют рассматривать процесс образования отдельных зерен и их роста.

Помимо аналитических моделей существует подход, заключающийся в имитации процесса зарождения и роста на основе модели И.Л. Миркина, в которой предлагается изучать процесс кристаллизации с помощью двухмерных схем [6].

В работах $[7,8]$ авторами развита диффузионная модель превращения аустенита, основанная на использовании физических принципов термодинамики. Полученные уравнения для скорости образования перлита и 
Ministry of Education and Science of Ukraine

The National Metallurgical Academy of Ukraine, Dnipro, 17 - 19 March, 2020

межпластиночного расстояния от величины переохлаждения с достаточной степенью соответствуют экспериментальным данным для углеродистых сталей.

Общим недостатком описанных выше работ является пренебрежение влиянием напряженно-деформированного состояния, возникающего в процессе термообработки деталей, на кинетику структурных превращений, в то время как имеющиеся экспериментальные данные свидетельствуют о существенном влиянии напряжений и деформаций на структурные превращения.

Целью настоящей работы является сравнение аналитических моделей распада аустенита с учетом внутренних напряжений, полученных различными авторами.

Результаты исследований. Напряжения при термической обработке стальных изделий возникают вследствие объемных деформаций, связанных с неравномерностью температурного поля и неодновременностью протекания структурных превращений. Причем деформации, обусловленные структурными переходами, соизмеримы с температурными деформациями [9]. Описание температурных и структурных деформаций обычно проводится с помощью суммарного коэффициента линейного расширения, равного [10]:

$$
a=d \varepsilon / d t
$$

где $\varepsilon$ - свободная деформация, учитывающая температурные и структурные изменения. Значения $\varepsilon$ можно определить по дилатограмме [10] стали, в зависимости от структурного состава, исходя из правила аддитивности [11].

В работе [12] предложена математическая модель закалки стали с учетом влияния напряжений на перлитное и мартенситное превращения. Для описания перлитного превращения в неизотермических условиях использована теория изокинетических реакций [5]. Принято, что ход перлитного превращения зависит от среднего напряжения. При описании изотермической кинетики по уравнению Аврами предложено заменить коэффициент К следующим выражением:

$$
\mathrm{Ka}=\mathrm{K}(\mathrm{t}) \exp \left(\mathrm{nc} \sigma_{0}\right),
$$


International scientific and technical conference Information Technologies in Metallurgy and Machine building - ITMM 2020

где $\sigma_{0}$ - среднее напряжение [13]; с - экспериментально найденный коэффициент, t - температура стали.

Для мартенситного превращения принята зависимость от интенсивности напряжений, обобщающая формулу Коэстинена - Марбургера [19]:

$$
\mathrm{VM}=(1-\mathrm{Vp})\left\{1-\exp \left[-\mathrm{a}\left(\mathrm{Ms}-\mathrm{t}+\mathrm{b} \sigma_{\mathrm{i}}\right)\right]\right\},
$$

где $\sigma_{i}$ - интенсивность напряжений [13]; $a, b$ - константы, полученные экспериментально.

Основное допущение работы, заключающееся в том, что смещение области бейнитных превращений ИТД описывается так же, как для перлитной, соотношением (2), вряд ли оправдано. Бейнитное превращение, как и мартенситное, имеет сдвиговый характер [14], и поэтому для него, как и для мартенситного превращения [11], целесообразно принять зависимость кинетических параметров процесса от интенсивности напряжений. Такое предположение сделано в работе [15], в которой смещение ИТД под действием сложного напряженного состояния осуществлено по зависимостям, полученным для простого растяжения при замене $\sigma$ на $\sigma i$.

Авторами статьи [16] при исследовании ускорения хода мартенситного превращения под действием растягивающих напряжений, протекающего при отрицательных температурах в хромоникелевых сталях, сделано предположение, что кинетика распада зависит как от среднего напряжения, так и от интенсивности напряжений. Для мартенситного превращения использовано уравнение:

$$
\mathrm{VM}_{M}=\left(1-\mathrm{Vp}-\mathrm{V}_{\mathrm{B}}\right)\left\{1-\exp \left[-\mathrm{a}\left(\mathrm{M}_{\mathrm{s}}-\mathrm{t}+\mathrm{a}_{\mathrm{M}} \sigma_{0}+\mathrm{b}_{\mathrm{M}}^{\mathrm{i}} \sigma_{\mathrm{i}}+\mathrm{g}_{\mathrm{M}} \varepsilon_{\mathrm{i}}^{\mathrm{p}}\right)\right],\right.
$$

где $a_{M}, b^{i}{ }_{M}, g_{M}, a-$ эмпирические константы.

Вследствие отсутствия экспериментальных данных для определения многочисленных параметров математической модели, значения части констант положены равными нулю, другие приближенно приняты по работам [17].

В работе [18] теоретически найдено выражение, устанавливающее влияние уровня напряжений в $\gamma$-фазе на скорость образования мартенсита $d n_{\alpha} / d t:$

$$
d n_{\alpha} / d t=\left(L_{11} \Delta \phi+L_{12} \sigma_{\gamma}\right) e^{-\frac{v}{L} t}
$$


где $\Delta \varphi$ - термодинамическая сила для железа - изменение химического потенциала при переходе частиц из $\gamma$-фазы в $\alpha$-фазу, $\sigma_{\gamma}$-напряжение в $\gamma$-фазе; $L_{11}$ и $L_{12}$ - коэффициенты, зависящие от температуры превращения; $v$ - скорость распространения микродеформации в образце ( 1000 м/с); $L$ - характеристическое расстояние, на которое распространяется микродеформация сдвига (размер мартенситных реек или пластин). На начальном этапе образования структуры сдвига оно имеет величину порядка диаметра аустенитного зерна ( 100мкм), а затем при понижении температуры уменьшается [18].

Интегрируя скорость образования мартенсита, определяемую формулой (5) при постоянной температуре и времени от 0 до $\infty$, получаем следующее простое теоретическое выражение для расчета количества образующегося мартенсита в зависимости от напряжения в $\gamma$-фазе:

$$
\mathrm{n}_{\alpha}=\mathrm{n}_{\alpha 0}+\mathrm{L}_{12} \sigma_{\gamma}
$$

где $n_{\alpha 0}-$ количество мартенсита, которое образуется при отсутствии напряжений в $\gamma$-фазе. Это количество может быть рассчитано по формуле Коэстинена - Марбургера [19], или по более точной формуле, предложенной в работе [20]:

$$
n_{\alpha 0}=A_{m}\left(1-\exp \left(-K_{\alpha} \frac{M_{s}-T}{T-M_{f}}\right)\right.
$$

где $A_{m}$ - количество аустенита, сохранившегося до температуры $M_{s}$;

$M_{s}$ - температура начала образования мартенсита;

$M_{f}$ - температура конца образования мартенсита;

$K_{\alpha}$ - коэффициент, характеризующий скорость образования мартенсита вблизи температуры $M_{\text {s. }}$

Выводы. Накопленные к настоящему времени экспериментальные данные не позволяют достоверно описать влияние напряженного состояния на все структурные переходы при термообработке легированных сталей. Для надежного прогнозирования структурного состояния легированных сталей при термообработке необходимо проведение комплексного исследования, связанного с экспериментальным изучением влияния величины напряжений на скорость всех превращений и создание на базе полученных опытных 
International scientific and technical conference

Information Technologies in Metallurgy and Machine building - ITMM 2020

данных адекватной математической модели распада аустенита с учетом указанного влияния.

\section{Литература}

1. А.Н. Колмогоров. Известия АН СССР, серия математическая, 1937, №3, с. 355.

2. M. Avrami. Kinetics of phase change. I. General theory [J]. Journal of Chemical Physics, 1939, 7(12) P.1103-1112.

3. Физическое металловедение: Пер. с англ. Т.2. Фазовые превращения / Под ред. Р. Кана и П. Хаазена - М.: Металлургия, 1987. - 492с.

4. Б.Я. Любов. Кинетическая теория фазовых превращений. - М.: Металлургия,1969. 264c.

5. Дж. Кристиан. Теория превращений в металлах и сплавах. Ч.1. Термодинамика и общая кинетика. - М.: Мир, 1978. - 808с.

6. И.Л. Миркин. Исследование эвтектоидной кристаллизации стали // Структура и свойства сталей и сплавов: сб. тр. Моск. ин-та стали - М.: Оборонгиз, 1941. - С.5-158.

7. Бобырь С.В., Большаков В.И. Модели и характеристики прерывистого превращения аустенита в железоуглеродистых сплавах//Успехи физики металлов, 2014, Т.15, C.123-150.

8. Бобырь С.В., Большаков В.И. Анализ стационарного эвтектоидного превращения в углеродистой стали на основе положений неравновесной термодинамики //Металлофизика и новейшие технологии, 2016, т. 38, № 7, С.983-995.

9. Абрамов В.В. Напряжения и деформации при термической обработке стали. - К.: Вища школа, 1985. - 133с.

10. Р.К. Вафин, А.М. Покровский, В.Г. Лешковцев. Прочность термообрабатываемых прокатных валков. - М.: Изд-во МГТУ им. Н.Э. Баумана, 2004. - 264с.

11. Морозов Н.П. Производство и эксплуатация крупных опорных валков /Н.П. Морозов, В.А. Николаев, В.П. Полухин, А.М. Легун/ - М.: Металлургия, 1977. - 77с.

12. Wang Z-G., Inoue T. Analysis of temperature, structure and stress during queching // Journal of the Society Materials Science of Japan. - 1983. - №360 - P.991-1003.

13. В.Е. Лошкарев. Математическое моделирование процесса закалки с учетом влияния напряжений на структурные превращения в стали // МиТОМ, 1986. - № 1. C.86-69.

14. Khan S.A., Bhadeshia H.K.D.H. The bainite transformation in chemically heterogeneous 300M high-strenght steel // Met. Trans., 1990., Vol. 21a, P. 859-875.

15. Contribution des contraintes internes d'origine thermice dans le calcul de l'avancement des transformation de phase refroidissement continu d'un acier XC80 / S. Denis, C. Bas, 
F.M.B. Fernandes, A. Simon // Memoires et Etudes Scientifique Revue de Metallurgie. 1986. - Vol. 83. - №10. - P.533-541.

16. Tanaka K., Sato Y. Analysis of super-plastic deformation during isothermal martensitic transformation // Res. Mechanica, - 1986. - Vol.17. - №3. - P.241-252.

17. Bhadeshia H.K.D.H. Some phase transformation in steel // Mater. Sci. and Technol., 1999, Vol. 15, P. 22-29.

18. Bobyr S.V. Non-equilibrium thermodynamic model austenitic non-diffusion transformation in iron-based alloys. / Machines. Technologies. Materials. International Scientific Journal, 2018., Is. 10, P.415-418.

19. Christian J.W. The Theory of Transformations in Metals and Alloys, 1st Edition, Pergamon Press, Oxford, UK, 2002.

20. Krot P.V., Bobyr S.V., Dedik M.O. Simulation of backup rolls quenching with experimental study of deep cryogenic treatment / Int. J. of Microstructure and Materials Properties, 2017, Vol.12, No.3/4. - P. 259- 275.

\section{ANALYTICAL MODELS OF AUSTENITE TRANSFORMATION IN STEEL WITH ALLOWANCE FOR INTERNAL STRESSES}

Bobyr Serhii, Borysenko Andrii, Loshkarev Dmytro

Abstract. The analysis of the presented works shows that mathematical modeling of austenite decomposition during heat treatment is possible using both TTT and CCT diagrams. The effect of the stress-strain state arising in the process of heat treatment of parts on the kinetics of structural transformations is usually not taken into account. The experimental data accumulated to date do not reliably describe the effect of the stress state on structural transitions during the heat treatment of steels. A simple theoretical expression is proposed for calculating the amount of martensite formed as a function of the stress in the $\gamma$-phase. To reliably predict the structural state of steels during hot deformation and heat treatment, it is necessary to conduct a comprehensive study related to the experimental study of the effect of stresses on the transformations of all types and the creation of an adequate mathematical model of austenite decomposition based on the experimental data obtained, taking into account this effect.

Keywords: AUSTENITE TRANSFORMATION, MARTENSITE, ANALYTICAL MODELS, INTERNAL STRESSES, HEAT TREATMENT. 


\section{References}

1. A.N. Kolmogorov. Izvestiya AN SSSR. seriya matematicheskaya. 1937. №3. s. 355.

2. M. Avrami. Kinetics of phase change. I. General theory [J]. Journal of Chemical Physics. 1939. 7(12) P.1103-1112.

3. Fizicheskoye metallovedeniye: Per. s angl. T.2. Fazovyye prevrashcheniya / Pod red. R. Kana i P. Khaazena - M.: Metallurgiya. 1987. - 492s.

4. B.Ya. Lyubov. Kineticheskaya teoriya fazovykh prevrashcheniy. - M.: Metallurgiya.1969. $264 s$.

5. Dzh. Kristian. Teoriya prevrashcheniy v metallakh i splavakh. Ch.1. Termodinamika i obshchaya kinetika. - M.: Mir. 1978. - 808s.

6. I.L. Mirkin. Issledovaniye evtektoidnoy kristallizatsii stali // Struktura i svoystva staley i splavov: sb. tr. Mosk. in-ta stali - M.: Oborongiz. 1941. - S.5-158.

7. Bobyr S.V.. Bolshakov V.I. Modeli i kharakteristiki preryvistogo prevrashcheniya austenita v zhelezouglerodistykh splavakh//Uspekhi fiziki metallov. 2014. T.15. S.123-150.

8. Bobyr S.V.. Bolshakov V.I. Analiz statsionarnogo evtektoidnogo prevrashcheniya v uglerodistoy stali na osnove polozheniy neravnovesnoy termodinamiki //Metallofizika i noveyshiye tekhnologii. 2016. t. 38. № 7. S.983-995.

9. Abramov V.V. Napryazheniya i deformatsii pri termicheskoy obrabotke stali. - K.: Vishcha shkola. 1985. - 133s.

10. R.K. Vafin. A.M. Pokrovskiy. V.G. Leshkovtsev. Prochnost termoobrabatyvayemykh prokatnykh valkov. - M.: Izd-vo MGTU im. N.E. Baumana. 2004. - 264s.

11. Morozov N.P. Proizvodstvo i ekspluatatsiya krupnykh opornykh valkov /N.P. Morozov. V.A. Nikolayev. V.P. Polukhin. A.M. Legun/ - M.: Metallurgiya. 1977. - 77s.

12. Wang Z-G., Inoue T. Analysis of temperature, structure and stress during queching // Journal of the Society Materials Science of Japan. - 1983. - №360 - P.991-1003.

13. V.E. Loshkarev. Matematicheskoye modelirovaniye protsessa zakalki s uchetom vliyaniya napryazheniy na strukturnyye prevrashcheniya v stali // MiTOM. 1986. - № 1. - S.86-69.

14. Khan S.A., Bhadeshia H.K.D.H. The bainite transformation in chemically heterogeneous 300M high-strenght steel // Met. Trans., 1990., Vol. 21a, P. 859-875.

15. Contribution des contraintes internes d'origine thermice dans le calcul de l'avancement des transformation de phase refroidissement continu d'un acier XC80 / S. Denis, C. Bas, F.M.B. Fernandes, A. Simon // Memoires et Etudes Scientifique Revue de Metallurgie. 1986. - Vol. 83. - №10. - P.533-541.

16. Tanaka K., Sato Y. Analysis of super-plastic deformation during isothermal martensitic transformation // Res. Mechanica, - 1986. - Vol.17. - №3. - P.241-252. 
17. Bhadeshia H.K.D.H. Some phase transformation in steel // Mater. Sci. and Technol., 1999, Vol. 15, P. 22-29.

18. Bobyr S.V. Non-equilibrium thermodynamic model austenitic non-diffusion transformation in iron-based alloys. / Machines. Technologies. Materials. International Scientific Journal, 2018., Is. 10, P.415-418.

19. Christian J.W. The Theory of Transformations in Metals and Alloys, 1st Edition, Pergamon Press, Oxford, UK, 2002.

20. Krot P.V., Bobyr S.V., Dedik M.O. Simulation of backup rolls quenching with experimental study of deep cryogenic treatment / Int. J. of Microstructure and Materials Properties, 2017, Vol.12, No.3/4. - P. 259- 275. 